\title{
Response to SARS-Covid-19-related visitor restrictions on labor and delivery wards in New York City
}

\author{
Alison Hermann ${ }^{1} \cdot$ Kristina M. Deligiannidis $^{2,3,4} \cdot$ Veerle Bergink $^{5} \cdot$ Catherine Monk $^{6} \cdot$ Elizabeth M. Fitelson $^{7}$. \\ Thalia K. Robakis $^{8} \cdot$ Catherine Birndorf $^{9}$
}

Received: 2 April 2020 / Accepted: 7 April 2020 / Published online: 15 April 2020

(C) Springer-Verlag GmbH Austria, part of Springer Nature 2020

Dear MONA members and reproductive mental health colleagues,

We are writing as leaders of reproductive mental health programs in the greater New York City metropolitan area, which has become the epicenter of the SARS-Covid19 pandemic in the USA. Recently many of our local hospitals have instituted a visitor restriction policy for labor and delivery services that does not allow a support person, including partners, to be physically present in the hospital. Many of you have expressed concern about potential psychological harms of these policies, which we understand and to which we would like to respond, especially as the visitor restrictions in NY hospitals soon may be necessary in other hospitals across the country.

We support the very difficult decision our hospital administrators have made to institute this visitor restriction policy. We are in a state of emergency and are grappling with the largest public health threat we have seen in our country in over a century. As of this writing, there are over 30,000 confirmed cases in our area, increasing by a factor of 2 every 3 days. Nearly a quarter of hospitalized patients require an ICU level

This article is part of the Topical Collection on Women's mental health during the Covid-19 pandemic Edited by: Anita Riecher-Rössler

Alison Hermann

alh9039@med.cornell.edu

1 Payne Whitney Women's Program, Weill Cornell Medicine-New York Presbyterian Hospital, New York, NY, USA

2 Women's Behavioral Health, Zucker Hillside Hospital, Queens, NY, USA

3 Psychiatry and Obstetrics \& Gynecology, Zucker School of Medicine at Hofstra/Northwell, Hempstead, NY, USA

4 Center for Psychiatric Neuroscience, Feinstein Institutes for Medical Research, Manhasset, NY, USA of care. New York City and its healthcare institutions are doing everything possible to care for these critically ill patients and overall increased health care needs, which is requiring them to take extraordinary measures such as erecting tents as makeshift emergency department expansions and repurposing spaces internally and throughout the city as emergency field hospitals and ICUs. In our state of New York, over 40,000 healthcare workers (some retired) have signed up to volunteer to be trained and/or redeployed to be part of the surge healthcare force. The scope of this crisis was unimaginable just weeks ago.

Personal protective equipment (PPE) will soon become in such short supply that unprecedented measures are being put into place to reduce the number of people needing to use it and to reuse it where ever it can be safely done. Staff in the hospital are being minimized to the extent possible, including removing medical students and others from the wards. Microbiology and engineering departments in our academic centers are racing to find ways to increase manufacturing of PPE supplies and to effectively sterilize and re-use them. Despite all of this, many our front-line healthcare workers

5 Women's Mental Health Program, Department of Psychiatry, Department of Obstetrics, Gynecology and Reproductive Science, Icahn School of Medicine at Mount Sinai, New York, NY, USA

6 Departments of Obstetrics and Gynecology, Women's Mental Health $@$ Ob/Gyn Columbia University Medical Center, New York State Psychiatric Institute, New York, NY, USA

7 The Women's Program, Columbia University Department of Psychiatry, New York, NY, USA

8 Women's Mental Health Program, Icahn School of Medicine at Mount Sinai, New York, NY, USA

9 The Motherhood Center of New York, Weill Cornell Medicine - New York Presbyterian Hospital, New York, NY, USA 
are returning to their shifts every day with the most limited of supplies and resources. Every day we receive more urgent requests from our administrators for PPE conservation. Unfortunately, not having enough PPE even for our most essential healthcare workers, we clearly do not have enough PPE to gown, mask, and shield visitors appropriately.

Some of our hospitals have already started to care for women in labor who have active Covid-19 infection. Detection of Covid-19 infection in this population has been a challenge as the presentation can be atypical and overlap with normal symptoms of pregnancy and of labor specifically. Because of these challenges, women in labor are now being universally tested for Covid-19 in several hospitals. However, testing for Covid-19 has also been a massive challenge as swabs to collect the sample are in very short supply, laboratory materials to analyze results are not scaled to meet the need, and time to get a result is on average $8-10 \mathrm{~h}$. Unfortunately, we do not have the capacity to additionally test visitors including partners, who may have asymptomatic or very mild symptoms yet spread the virus to the baby as well as hospital personnel.

Given the frequency of infection, lack of PPE, and shortage of testing, at this time we cannot effectively mitigate the risk of Covid-19 transmission that visitors pose to healthcare workers and other patients including neonates. We must do what we can to ensure that the SARS-Covid-19 virus does not cross the threshold of our NICUs and nurseries. We must additionally do what we can to minimize occupational exposure of front-line healthcare workers as illness or quarantine procedures for this population render them unavailable for future patients' deliveries.

As reproductive mental health experts, we are wellpracticed at making difficult decisions in high-stakes situations where evidence is limited. We understand the "risk-risk" framework and the uncomfortable reality that there are circumstances in which potential harms, as well as our ability to mitigate them, must be weighed against each other. Applying those principles here, it is our assessment that the public health risk of SARS-Covid-19 transmission on labor and delivery wards from the presence of visitors, including partners, is greater and less modifiable than the risk of psychological harms that come from this physical separation.

Given much collective experience working with laboring women, including those who have been separated from partners under different circumstances such as military deployments, we believe we in the Marce community can offer meaningful strategies to mitigate the risk of psychological harms from this visitor restriction policy and to increase resilience in these patients. In tandem with announcing the visitor restriction policy, many of our hospitals are adjusting staffing to ensure that patients admitted to L\&D services have greater access to nursing and other supports. We are maximizing the use of technology where ever it is feasible and appropriate to maintain social contact with loved ones and supporters despite the physical separation. We are also developing creative liaison models to extend the reach of mental health services to these patients and to reduce burn out and trauma in front-line health care workers who are caring for them. In addition, we are advocating internally and more broadly for this policy to be in place only for the minimum amount of time necessary and for it to be revisited promptly as both risk and mitigation circumstances change.

At this point, we ask you to discuss and plan for how the pandemic may soon affect the clinical care of perinatal women in your region. It is our shared mission to continue to care for the mental health needs of our perinatal patients, no matter what the circumstances. We believe that in writing this letter we may help inform other areas throughout the USA who we expect will find themselves in a similar situation within a matter of days to weeks. We urge you to start planning now. This experience has confronted us with an unfortunate expertise that we are hoping to harness for the benefit of all. We very much appreciate having this community and the power it has to do so much good. Please join us.

\section{Compliance with ethical standards}

Conflict of interest Dr. Hermann served as a consultant for Sage Therapeutics and is a cofounder and chief medical officer of Iris OB Health, Inc. Dr. Deligiannidis receives research grant support from NIMH, Sage Therapeutics and Vorso Corporation, serves as a consultant to Sage Therapeutics, and receives royalties from an NIH Employee Invention. Dr. Robakis receives research grant support from Vorso Corporation. Dr. Birndorf is CEO of The Motherhood Center of New York.

Ethical approvals Not applicable (this was not a research study).

Informed consent Not applicable (no research subjects).

Publisher's note Springer Nature remains neutral with regard to jurisdictional claims in published maps and institutional affiliations. 